DOI: $10.12731 /$ wsd-2018-3-62-76

УДК 616.831-06: 616.89.88+159.98: 61

\title{
НЕЙРОПСИХОЛОГИЧЕСКАЯ ХАРАКТЕРИСТИКА КОГНИТИВНЫХ НАРУШЕНИЙ ПРИ ПРЕОБЛАДАНИИ ЛОБНОЙ СИМПТОМАТИКИ В СТРУКТУРЕ ДЕМЕНЦИИ В ПОЗДНЕМ ВОЗРАСТЕ
}

\author{
Ефименко Т.С., Локтева Д.Н., Перевощикова А.А.
}

Цель: изучить нейропсихологические аспекты когнитивных нарушений у пациентов с преобладанием лобной симптоматики в сравнении с когнитивными нарушениями у паџиентов сенильной деменщией иной структуры.

Материалы и методы. Обследовано 48 человек, страдаюших сенильной деменцией. Основную группу с преобладанием лобной симптоматики составили 24 больныхх, из них 12 (50\%) женщины, средний возраст $84 \pm 12$ лет. Контрольную группу с иной структурой сенильной деменции также составили 24 больных, из них 12 (50\%) женщины, средний возраст $-83 \pm 13$ лет.

Исследование проводилось с разрешения этического комитета ФГБОУ ВО ЮУГМУ. Каждый участник (его законный представитель) подписывал стандартную форму добровольного согласия для участия в медициском исследовании.

Использовались Батарея тестов для оиенки лобной дисфункиии (БЛД); Краткая шкала оченки психического статуса (MMSE); Монреальская шкала оценки когнитивных функций (МоСА); Схема нейропсихологического обследования Е.Д. Хомской: пробы на исследование праксиса, речи, слухо- и зрительно-моторного гнозиса.

Непараметрическое сравнение результатов проводилось при помощи критерия Манна-Уитни, параметрическое - при помощи углового преобразования Фишера (УПФ) в среде статистической программы SPSS v.20.

Результаты. Констатирующий эксперимент раскрыл характер нейропсихологических паттернов когнитивных нарушений, свойственных больнымм сенильной деменцией с преобладанием лобной симптоматики в клинической картине расстройства. Выявлены сравнительные особен- 
ности влияния лобной симптоматики на характер когнитивных нарушений в структуре сенильной деменции. Сформированы предпосылки для модернизации существующих программ реабилитащии данной категории больных.

Заключение. Таким образом, выявлены предпосылки для динамической оиенки эффективности лечебно-реабилитационных мероприятий у больных сенильной деменщией с преобладанием лобной симптоматики.

Ключевые слова: сенильная деменция; лобная симптоматика; когнитивные нарушения.

\title{
NEUROPSYCHOLOGY OF COGNITIVE DYSFUNCTIONS IN SENILE DEMENTIA WITH LOBAR SYMPTOMS PREVALENCE
}

\author{
Efimenko T.S., Lokteva D.N., Perevoschikova A.A.
}

Background: comparative cognitive dysfunctions in lobar and non-lobar senile dementia patients (constitute experiment).

Materials \& Methods: 48 senile dementia patients, equal 24 in lobar $(84 \pm 12$ mean age) and non-lobar ( $83 \pm 13$ mean age) groups, 50\% female. Ethic committee granted. MMSE, MoCa, Lobar dysfunction battery (LDB), E. Homskaya probes (praxis, speech, audio- and visual motor gnosis) were used. Mann-Whitney and Fisher criteria for comparison in SPSS v. 20 were used.

Results: constitute experiment presents neuropsychological patterns of cognitive dysfunctions in senile dementia patients with lobar symptoms prevalence. Comparative measures of lobar induction on cognitive dysfunctions are discussed.

Conclusion: thus, even if a non-statistically meaning year of life is The Factor of senile dementia (and we'll try to equalize it in process of further investigations), the basis for dynamic research of efficacy of treatment and rehabilitation of this patients is grounded.

Keywords: senile dementia; lobar symptoms; cognitive dysfunction.

Актуальность исследования обусловлена увеличением продолжительности жизни населения Российской Федерации и необходимостью решения задач сохранения активного долголетия и повышения его качества в позднем возрасте [5]. По данным Национального руководства по 
неврологии (ред. Гусев Е.И., 2009), риск развития деменции среди лиц, страдающих когнитивными нарушениями, составляет до $15 \%$ в год [4]. В мае 2017 ВО3 приняла «Глобальный план действий по предотвращению деменций» на 2017-2025 годы. Коммюнике содержит сведения, что ежегодно деменцией заболевает 10 млн. человек, из них 6 млн. - в странах с неразвитой экономикой. Мероприятия в рамках плана нацелены не только на информирование населения и профилактику деменций, но и на осуществление исследований в данной области [3]. Деменции «лобного» типа, включая специфичную лобно-височную дегенерацию, распространены значительно меньше, чем болезнь Альцгеймера $[11,12]$, однако в связи с ростом продолжительности жизни, расчётный риск увеличивается с каждым годом [2]. Однако не исключено, что данной проблематике до сих пор уделялось недостаточно внимания, и показатели распространённости известны не на уровне реальной популяции. Основными симптомами поражения лобных долей мозга признаются нарушения управляющих функций [7], которых, выделяют четыре: когнитивные, связанные с целенаправленностью, планированием и контролем оперантной деятельности; поведенческие, связанные с эмоциональным подкреплением саморегуляции операнты; регулирующие субъектную активность в постановке целей и принятия результатов; метакогнитивные процессы, связанные с мотивацией достижения. Нарушение функций лобных долей приводит к распаду сложных программ деятельности и их замене сначала более простыми «полевыми» формами поведения, а затем инертными стереотипами, потерявшими связь с ситуацией. Следствием нарушений управляющих функций становятся нарушения критичности, и, в дальнейшем, распад эмоционально-личностной сферы и высших психических функций [6].

В последние 30 лет странами с наибольшей продолжительностью жизни (Япония, Южная Корея, южные регионы КНР, Австралия и страны Трансатлантического союза) накоплен обширный пул сведений по данной проблематике. Стали известны причины заболевания. Z. Tong et al [16]. называют причиной когнитивного дефицита у больных сенильной деменцией нарушения обмена веществ, приводящие к повышению уровней эндогенного формальдегида с формированием амилоида в белом веществе мозга. Вместе с тем, фармакологический прорыв оказался невозможен. A.D. Korczyn [13] отмечает, что, не смотря на прорыв в понимании причин сенильной деменции альцгеймеровского типа, не удалось найти терапевтический подход к снижению уровня амилоида в головном мозге. 
Автор предлагает сосредоточить усилия на профилактике факторов риска заболевания в среднем возрасте. Продолжается изучение клиники расстройства. Так, H.V. Vinters [18], обсуждая патогенез болезни Альцгеймера и сенильной деменции альцгеймеровского типа, отмечает когнитивное снижение как главную проблему самых пожилых больных. C. Ballard et al [8]. сравнили выраженность когнитивного снижения у больных тремя типами деменции, выявив наилучшие результаты у больных сенильной деменцией с тельцами Леви. Отмечено, что когнитивное снижение у женщин было более выражено в базовом эксперименте, однако нивелировалось в течение года динамического наблюдения. Тем самым показано статистически незначимое значение года жизни с сенильной деменцией. A.J. Gray et al [10]. изучили у больных сенильной деменцией альцгеймеровского типа в сравнении с больными сосудистой деменцией возможности обоняния, и обнаружили, что восприятие запахов страдает при этом расстройстве так же, как и другие перцептивные процессы.

Проблемной сферой у данной категории больных являются не только лечение и реабилитация, но и сама диагностика. Так, G.R. Tack et al [15]. отмечают, что ведущей проблемой для улучшения технологий мониторинга состояния больных сенильной деменцией являются нарушения восприятия. Сложности представляет и дифференциально-диагностический процесс в позднем возрасте. Так, Е.H. Rubin et al [14]. показали, что больные униполярной депрессией в позднем возрасте выполняют психометрические тесты сходно с больными умеренной деменцией альцгеймеровского типа, что требует бережной дифференциальной диагностики. А.А. Атаманов [1] также отмечал признаки псевдодементного поведения у больных генерализованным тревожным расстройством в пресениуме.

Существенным препятствием для терапии и реабилитации остаётся летальная реакция человека в позднем возрасте практически на любые стимулы и стрессоры. Как отмечают K. Ukai, Y. Mizuno [17], за год в гериатрическом отделении у больных сенильной деменцией случилось 56 эпизодов угрожающих жизни состояний, 6 пациентов погибло. Преимущественно это пневмония и обструкция верхних дыхательных путей, но также и ломкость костей и онкопатология. Наиболее оптимистично к проблеме настроены представители альтернативной неевропейской медицины. Так, H. Yan et al [19]. делятся успешным опытом лечения сенильной деменции методами традиционной китайской медицины, сочетающей лекарственные средства, физиотерапию и лечебную гимнастику. 
Наибольшую социальную проблему представляет забота о таких больных. I. Fukunishi, K. Hosokawa [10] изучили психологическое состояние семей, в которых проживают больные сенильной деменцией. Наибольшие проблемы невротического и депрессивного спектра отмечены у родственников, ухаживающих за больным сенильной деменцией в одиночку. Авторы делают вывод о необходимости большего вовлечения социальных и медицинских служб в оказание помощи этой категории больных на дому. В свете программы ВОЗ по повышению качества жизни больных деменцией, щадящие программы реабилитации должны включать и микросоциальное окружение больного.

Исследования «лобных» деменций как маргинального этапа развития деменций альцгеймеровского типа в настоящее время являются высоко актуальными.

Цель настоящей работы - изучить нейропсихологические аспекты когнитивных нарушений у пациентов с преобладанием лобной симптоматики в сравнении с когнитивными нарушениями у пациентов сенильной деменцией иной структуры.

\section{Материалы и методы.}

Обследовано 48 человек, страдающих сенильной деменцией. Основную группу с преобладанием лобной симптоматики составили 24 боль-

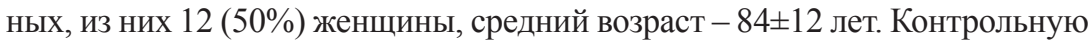
группу с иной структурой сенильной деменции также составили 24 боль-

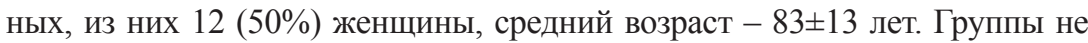
различались по полу, возрасту и уровню образования.

Исследование проводилось с разрешения этического комитета ФГБОУ ВО ЮУГМУ Минздрава России. Каждому участнику (его законному представителю в подавляющем большинстве случаев) разъяснялась суть эксперимента в стандартной письменной форме с указанием плана исследования, после чего участник подписывал стандартную форму добровольного согласия для участия в медицинском исследовании.

Клинико-психологическому исследованию предшествовало изучение медицинской документации: заключений специалистов - невролога, психиатра; данных электроэнцефалографии (ЭЭГ) и компьютерной томографии (КТ), наблюдение и беседа с каждым больным. Использовались Батарея тестов для оценки лобной дисфункции (БЛД). Результат менее 12 баллов свидетельствовал о лобной дисфункции; Краткая шкала оценки психического статуса (MMSE) в сфере интеллектуально-мнестических 
нарушений. Результат менее 25 баллов указывал на наличие деменции; Монреальская шкала оценки когнитивных функций (МоСА). О наличии деменции также свидетельствовал результат менее 25 баллов; Схема нейропсихологического обследования Е.Д. Хомской. Применялись пробы на исследование праксиса, речи, слухо- и зрительно-моторного гнозиса. Результаты оценивались по 3-х бальной шкале: 1 - нет нарушений, 2 - слабо выраженные нарушения, 3 - сильно выраженные нарушения.

Непараметрическое сравнение результатов проводилось при помощи критерия Манна-Уитни, параметрическое - при помощи углового преобразования Фишера (УПФ) в среде SPSS v. 20.

Результаты исследования подтвердили поверхностно очевидную выраженность когнитивного дефицита у больных с преобладанием лобной симптоматики в структуре деменции, однако отдельные нейропсихологические аспекты не могут не вызывать интерес.

Таблица 1.

Результаты сравнения статистической тяжести случая при наличии и отсутствии лобной симптоматики в структуре деменции

\begin{tabular}{|l|c|c|c|}
\hline Шкала & Основная группа (M $\pm \mathbf{m})$ & Контрольная группа $(\mathbf{M} \pm \mathbf{m})$ & $\mathbf{p}=$ \\
\hline БЛД & $8,54 \pm 2,5$ & $\mathbf{1 4 , 5 4} \pm \mathbf{1 , 5}$ & 0,000 \\
\hline MMSE & $17,71 \pm 4,0$ & $\mathbf{2 1 , 4 2 \pm 3 , 0}$ & 0,001 \\
\hline MоCA & $12,46 \pm 4,4$ & $\mathbf{1 7 , 2 5} \pm \mathbf{3 , 3}$ & 0,000 \\
\hline
\end{tabular}

Таблища 2.

Результаты сравнения выраженности лобной симптоматики в структуре деменции

\begin{tabular}{|l|c|c|c|}
\hline \multicolumn{1}{|c|}{ Показатель } & \multicolumn{1}{c|}{$\begin{array}{c}\text { Основная } \\
\text { группа }\end{array}$} & $\begin{array}{c}\text { Контрольная } \\
\text { группа }\end{array}$ & $\mathbf{p}=$ \\
\hline Обобщение & 0,79 & $\mathbf{2 , 4 2}$ & 0,000 \\
\hline Беглость речи & 1,08 & $\mathbf{2 , 2 5}$ & 0,000 \\
\hline Динамический праксис & 1,62 & $\mathbf{2 , 3 7}$ & 0,001 \\
\hline Простая реакция выбора & 1,29 & $\mathbf{2 , 5 4}$ & 0,000 \\
\hline $\begin{array}{l}\text { Усложненная реакция } \\
\text { выбора }\end{array}$ & 0,83 & $\mathbf{1 , 9 6}$ & 0,000 \\
\hline Хватательный рефлекс & 2,96 & 2,92 & 0,33 \\
\hline
\end{tabular}

Как следует из таблицы 1 , средние статистические показатели основной группы по всем характеристикам лобной и когнитивной дисфункции были достоверно $(\mathrm{p} \leq 0,001)$ грубо снижены в сравнении с контролем. Ниже в 
таблице 2 приведены данные Батареи тестов лобной дисфункции (БЛД), свидетельствующие о достоверно $(\mathrm{p} \leq 0,001)$ крайнем дефиците способности к обобщению, беглости речи, простой и усложненной реакции выбора, грубом страдании динамического праксиса. Не различалась с контролем только сохранность хватательного рефлекса. При проведении исследований больные наличие дефекта критически не оценивали, сохраняли эйфоричный или апатичный эмоциональный фон, на усталость не жаловались, по завершении уходили неохотно, задерживая сопровождающих.

Ниже в таблице 3 представлено распределение изученных случаев по степени тяжести проявлений сенильной деменции в зависимости от наличия лобной симптоматики. Больные основной группы не были чувствительны к методике выявления когнитивных нарушений, в то время как больные контрольной группы показывали лучшие результаты по методике MMSE, проводившейся за день до МоСА.

Таблица 3.

Результаты сравнения статистической степени тяжести расстройства при наличии и отсутствии лобной симптоматики в структуре деменции

\begin{tabular}{|l|c|c|c|c|c|c|}
\hline $\begin{array}{c}\text { Степень } \\
\begin{array}{c}\text { выраженности } \\
\text { деменции } \\
\text { в баллах }\end{array}\end{array}$ & $\begin{array}{c}\text { Основ- } \\
\text { ная } \\
\text { группа } \\
\mathbf{( \% )}\end{array}$ & $\begin{array}{c}\text { Кон- } \\
\text { троль- } \\
\text { ная } \\
\text { группа } \\
\mathbf{( \% )}\end{array}$ & $\begin{array}{c}\text { Итого } \\
\mathbf{( \% )}\end{array}$ & $\begin{array}{c}\text { Основ- } \\
\text { ная } \\
\text { группа } \\
\mathbf{( \% )}\end{array}$ & $\begin{array}{c}\text { Кон- } \\
\text { троль- } \\
\text { ная } \\
\text { группа } \\
\mathbf{( \% )}\end{array}$ & $\begin{array}{c}\text { Итого } \\
\mathbf{( \% )}\end{array}$ \\
\hline Предеменция (>26) & 0 & 4,2 & 4,2 & 0 & 0 & 0 \\
\hline Легкая (20-25) & 0 & 16,6 & 16,6 & 0 & 16,6 & 16,6 \\
\hline Умеренная (11-19) & $\mathbf{3 3 , 4 ^ { * }}$ & 29,2 & 62,6 & 33,4 & 31,3 & 64,7 \\
\hline Тяжелая (<10) & 16,6 & 0 & 16,6 & $\mathbf{1 6 , 6 *}$ & 2,1 & 18,7 \\
\hline Всего & 50 & 50 & 100 & 50 & 50 & 100 \\
\hline
\end{tabular}

*Примечание. Различие достоверно $(\mathrm{p}<0,05)$.

В целом, в контрольной группе первое общение с клиническим психологом и тест БЛД вызывали неподдельный интерес, MMSE проходил «на подъёме», с высоким уровнем неформального общения и желанием задержаться подольше. МоСА проходил ровно, с нервозностью в ряде случаев. А исследование по Холмской - либо более нервно, либо более равнодушно. Расставание в большинстве случаев не сопровождалось желанием узнать результат. В основной группе общение было исключительно формальным, беспокойство вызывала только неспособность быстро 
усвоить инструкции, которые в дальнейшем использовались стереотипно, с затуханием.

Ниже в таблице 4 представлены сравнительные характеристики когнитивных нарушений по МоСА.

Таблийа 4.

Результаты сравнения когнитивных функций при наличии и отсутствии лобной симптоматики в структуре деменции

\begin{tabular}{|l|c|c|c|}
\hline \multicolumn{1}{|c|}{ Показатель } & $\begin{array}{c}\text { Основная } \\
\text { группа }\end{array}$ & $\begin{array}{c}\text { Контроль- } \\
\text { ная группа }\end{array}$ & $\mathbf{p}=$ \\
\hline Оптико-пространственная деятельность & $\mathbf{0 , 5}$ & 1,67 & 0,001 \\
\hline Называние & $\mathbf{2 , 4 6}$ & 2,88 & 0,04 \\
\hline Внимание (ряд цифр) & 1,29 & 1,67 & 0,12 \\
\hline Внимание (ряд букв) & $\mathbf{0 , 4 6}$ & 0,92 & 0,000 \\
\hline Внимание (счет) & $\mathbf{0 , 7 1}$ & 1,54 & 0,009 \\
\hline Повторная речь & 0,54 & 0,96 & 0,13 \\
\hline Беглость речи & 0,21 & 0,42 & 0,10 \\
\hline Абстрактное мышление & 0,58 & 0,96 & 0,06 \\
\hline Память & 0,5 & 0,83 & 0,32 \\
\hline Ориентировка в месте и времени & $\mathbf{4 , 9 2}$ & 5,71 & 0,05 \\
\hline
\end{tabular}

Наиболее грубо у больных с преобладанием лобной симптоматики в структуре деменции страдали оптико-пространственная деятельность и внимание при выделении ряда букв и вычитании из 100 по 7, что указывает на страдание программных операций. По той же причине были достоверно $(\mathrm{p} \leq 0,05)$ снижены называние и ориентировка в месте и времени. Следует отметить, что по глубине поражения беглости речи и абстрактного мышления группы не различались, хотя ожидалось, что эти показатели в группе «лобных» больных «должны быть» значительно хуже. Можно утверждать, что «лобные» деменции также обладают какими-то возможностями компенсации, что требует дальнейшего изучения в интересах разработки реабилитационных программ для данной категории больных.

Ниже в таблице 5 приведены результаты исследования нарушений праксиса по схеме Е.Д. Хомской. Как следует из таблицы 5, в группе лобных деменций достоверно $(\mathrm{p}<0,05)$ были снижены результаты проб реципрокной координации, асимметричного постукивания, серийных программ и конструктивного праксиса, связанные с закономерным страданием конструктивных операций. При этом результаты остальных 10 проб между основной и контрольной группой не различались, что указывает на возможность ис- 
пользования уже существующих программ восстановления нарушенных функций праксиса в том числе и при «лобных» деменциях. Вместе с тем, необходимы дальнейшие исследования, направленные на модернизацию существующих реабилитационных программ в интересах «лобных» больных. Очевидно, что настало время преодоления демотивирующих стереотипов по отношению к «лобной» симптоматике в структуре деменции.

Таблица 5.

Результаты сравнения нарушений праксиса при наличии и отсутствии лобной симптоматики в структуре деменции

\begin{tabular}{|l|c|c|c|}
\hline \multicolumn{1}{|c|}{ Показатель } & $\begin{array}{c}\text { Основная } \\
\text { группа }\end{array}$ & $\begin{array}{c}\text { Контрольная } \\
\text { группа }\end{array}$ & $\mathbf{p}=$ \\
\hline Простые инструкции & 1,17 & 1,0 & 0,10 \\
\hline Праксис позы & 1,62 & 1,42 & 0,35 \\
\hline Пространственный праксис & 1,5 & 1,29 & 0,28 \\
\hline Проба Хэда & 1,54 & 1,29 & 0,23 \\
\hline Динамический праксис & 2,21 & 1,87 & 0,18 \\
\hline Реципрокная координация & $\mathbf{1 , 7 9}$ & 1,29 & 0,03 \\
\hline Асимметричное постукивание & $\mathbf{2 , 2 1}$ & 1,54 & 0,003 \\
\hline Серийные программы & $\mathbf{1 , 8 3}$ & 1,13 & 0,001 \\
\hline Конструктивный праксис & $\mathbf{2 , 0 8}$ & 1,42 & 0,001 \\
\hline Рисунок & 2,04 & 1,7 & 0,13 \\
\hline Предметные действия & 1,04 & 1,0 & 0,33 \\
\hline Движения взора & 1,08 & 1,0 & 0,33 \\
\hline Оральный праксис & 1,04 & 1,0 & 0,33 \\
\hline Условные реакции & 2,08 & 1,71 & 0,15 \\
\hline
\end{tabular}

Таблица 6.

Результаты сравнения нарушений речи при наличии и отсутствии лобной симптоматики в структуре деменции

\begin{tabular}{|l|c|c|c|}
\hline \multicolumn{1}{|c|}{ Показатель } & $\begin{array}{c}\text { Основная } \\
\text { группа }\end{array}$ & $\begin{array}{c}\text { Контрольная } \\
\text { группа }\end{array}$ & $\mathbf{p}=$ \\
\hline Спонтанная речь & 1,08 & 1,0 & 0,33 \\
\hline Автоматизированная речь & 1,04 & 1,0 & 0,33 \\
\hline Дезавтоматизированная речь & $\mathbf{1 , 9 6}$ & 1,21 & 0,000 \\
\hline Повторная речь & $\mathbf{1 , 7 9}$ & 1,13 & 0,002 \\
\hline Называние предметов & $\mathbf{1 , 2 9}$ & 1,0 & 0,049 \\
\hline Понимание речи & $\mathbf{1 , 5}$ & 1,21 & 0,02 \\
\hline Спонтанная развернутая речь & 1,33 & 1,08 & 0,11 \\
\hline
\end{tabular}


Как следует из таблицы 6 , достоверно $(\mathrm{p}<0,05)$ более грубые речевые нарушения в группе больных сенильной деменцией с преобладанием лобной симптоматики были обусловлены различными, но глубоко взаимосвязанными причинами. Страдание понимания речи и нарушения дезавтоматизированной речи непосредственно определялись программными нарушениями. Номинативные нарушения - динамической и/или моторной афазиями; расстройства повторной речи - стереотипиями. И динамическая афазия, и стереотипии имеют в своей основе все те же программные нарушения. Центральная для «лобной» деменции неэффективность речевого контакта в процессе реабилитации требует поиска иных решений.

Таблица 7.

Результаты сравнения нарушений слухового гнозиса при наличии и отсутствии лобной симптоматики в структуре деменции

\begin{tabular}{|l|c|c|c|}
\hline \multicolumn{1}{|c|}{ Показатель } & $\begin{array}{c}\text { Основная } \\
\text { группа }\end{array}$ & $\begin{array}{c}\text { Контрольная } \\
\text { группа }\end{array}$ & $\mathbf{p}=$ \\
\hline Звуковысотные отношения & 1,35 & 1,17 & 0,16 \\
\hline Узнавание мелодии & $\mathbf{1 , 9 1}$ & 1,52 & 0,04 \\
\hline Локализация звука в пространстве & 1,43 & 1,26 & 0,21 \\
\hline Дихотомический слух & 1,3 & 1,22 & 0,54 \\
\hline Повторение ритмов & $\mathbf{2 , 5 2}$ & 1,87 & 0,004 \\
\hline
\end{tabular}

Как следует из таблицы 7, у больных с преобладанием лобных нарушений в структуре деменции были достоверно $(\mathrm{p}<0,05)$ более выражены амузия - в силу программных расстройств, и нарушение повторения ритмов - в силу моторной стереотипии. Очевидно, что при выполнении слуховых невербальных заданий в рамках реабилитационной программы основное внимание должно уделяться различению звуковысотных соотношений и пространственной локализации источника звука, как более сохранным. Вместе с тем, в существующих реабилитационных программах упор делается как раз на распознавание мелодий и поддержание ритма. Данный подход нуждается в скорейшем пересмотре в интересах «лобных» больных и возможно более настойчивого вовлечения их в программы реабилитации.

Как следует из таблицы 8 , в рамках страдания зрительного гнозиса у больных с преобладанием лобных симптомов в структуре сенильной деменции были достоверно $(\mathrm{p}<0,05)$ более выражены нарушения, связанные с деструкцией программы действий - в наибольшей степени предметный гнозис и деление линий, в меньшей степени - зрительно-пространственный гнозис. Больные обеих групп в равной мере узнавали цвета, лица, 
цифры и буквы - различия в этих пробах достоверно $(\mathrm{p}<0,05)$ не различались. Следует заметить, что в быту и те, и другие охотно смотрят короткие мультфильмы, играют в несложные «двигательные» компьютерные игры, предпочитают совместное пребывание в условиях отделения одиночеству, вовлекаются в совместный досуг и участвуют в программах реабилитации. Адаптация и модернизация существующих реабилитационных программ навстречу потребностям, возможностям и способностям «лобных» больных - еще один шаг в развитии целого сектора здравоохранения.

Таблицуа 8.

Результаты сравнения нарушений зрительного гнозиса при наличии и отсутствии лобной симптоматики в структуре деменции

\begin{tabular}{|l|c|c|c|}
\hline \multicolumn{1}{|c|}{ Показатель } & $\begin{array}{c}\text { Основная } \\
\text { группа }\end{array}$ & $\begin{array}{c}\text { Контрольная } \\
\text { группа }\end{array}$ & $\mathbf{p}=$ \\
\hline Предметный гнозис & $\mathbf{1 , 9 6}$ & 1,13 & 0,000 \\
\hline Объем зрительного восприятия & 1,25 & 1,08 & 0,21 \\
\hline Узнавание лиц & 1,75 & 1,5 & 0,28 \\
\hline Цветовой гнозис & 1,08 & 1,04 & 0,57 \\
\hline Зрительно-пространственный гнозис & $\mathbf{2 , 5}$ & 1,96 & 0,02 \\
\hline Деление линии & $\mathbf{1 , 7 1}$ & 1,17 & 0,009 \\
\hline Буквенный гнозис & 1,17 & 1,0 & 0,10 \\
\hline Цифровой гнозис & 1,08 & 1,04 & 0,66 \\
\hline
\end{tabular}

\section{Заключение.}

Возможно, правы оппоненты данной работы, утверждавшие, что статистически незначимый год жизни с деменцией (средний возраст основной группы $84 \pm 12$ лет, контрольной - 83 \pm 13 лет) имеет огромное клиническое значение. В дальнейших исследованиях мы постараемся добиться оптимальных соотношений поло-возрастных и образовательных характеристик изученных групп. Возможно, правы и те оппоненты данной работы, что указали на нецелесообразность противопоставления друг другу разных проявлений единого процесса патологического старения. В дальнейших исследованиях, по нашему убеждению, ярче проявятся клинические и клинико-психологические особенности «лобных» деменций. Наша работа следует в рамках задач ВОЗ по решению проблем деменции, и в условиях увеличения продолжительности жизни побуждает к поиску инновационных подходов к «привычным» и «устоявшимся» представлениям в данной области. Предполагаем использовать в разработке реабилитационных программ отечественный и зарубежный опыт компьютерных технологий 
обратной связи, дополненной реальности, а также модернизацию традиционных способов реабилитации больных деменцией. Исследования в данном направлении будут продолжены.

\section{Список литературы}

1. Атаманов А.А. Ремитирующие тревожные состояния как особый вариант генерализованного тревожного расстройства // Журнал неврологии и психиатрии им. С.С. Корсакова. М.: Медиа Сфера, 2008. №5. С. 19-24.

2. Васенина Е.Е. Современные представления о диагностике и лечении лобно-височной деменции / Е.Е. Васенина, Н.И. Верюгина, О.С. Левин // Актуальные вопросы геронтопсихиатрии. 2015. № 3. С. 26-28.

3. Всемирная организация здравоохранения. Глобальный план действий по предотвращению деменций на 2017-2025 гг. www.who.int/entity/mediacentre/news/releases/2017/dementia-triple-affected/ru/

4. Гусев Е.И. (ред). Неврология. Национальное руководство. М.: ГЭОТАР-Медиа, 2009. 1035 с.

5. Каменецкая М.И. Нарушение когнитивных функций при психических заболеваниях в позднем возрасте / Учебно-методическое пособие. Тула, 2013. 50 c. C. $29-33$.

6. Левин О.С. Современные подходы к диагностике и лечению смешанной деменции // Трудный пациент. 2014.Т.12. № 5. С. 2-6.

7. Фёрстл Х. Деменция / Ханс Фёрстл, Альфред Мелике, Клаус Вайхель. М.: МЕДпресс-информ, 2011. 639 с.

8. Ballard C, Patel A, Oyebode F, Wilcock G. Cognitive decline in patients with Alzheimer's disease, vascular dementia and senile dementia of Lewy body type // Age Ageing. 1996 May;25(3):209-13.

9. Fukunishi I, Hosokawa K. A study of psychological aspects of families living together with senile dementia // Jpn J Psychiatry Neurol. 1990 Mar;44(1):19-24.

10. Gray AJ, Staples V, Murren K, Dhariwal A, Bentham P. Olfactory identification is impaired in clinic-based patients with vascular dementia and senile dementia of Alzheimer type // Int J Geriatr Psychiatry. 2001 May;16(5):513-7.

11. Guekht A, Skoog I, Korczyn AD, et al. A Randomised, Double- Blind, Placebo-Controlled Trial of Actovegin in Patients with Post-Stroke Cognitive Impairment: ARTEMIDA Study Design // Dement. Geriatr. Cogn. Dis. Extra. 2013. V. 3. № 1. P. 459-467.

12. Jack CR, Knopman DS, Jagust WJ, Petersen RC, et al. Tracking pathophysiological processes in Alzheimer's disease: an updated hypothetical model of dynamic biomarkers // Lancet Neurology. 2013;12(2):207-16 
13. Korczyn AD. Why have we failed to cure Alzheimer's disease? // J Alzheimers Dis. 2012; 29 (2): 275-82. doi: 10.3233/JAD-2011-110359.

14. Rubin EH, Kinscherf DA, Grant EA, Storandt M. The influence of major depression on clinical and psychometric assessment of senile dementia of the Alzheimer type // Am J Psychiatry. 1991 Sep;148(9):1164-71.

15. Tack GR, Choi MH, Lee SJ. Prioritizing problem features in Korean patients with senile dementia for implementation of monitoring technologies // Psychiatry Res. 2011 May 30;187(3):418-23. doi: 10.1016/j.psychres.2010.06.031. Epub 2010 Jul 24.

16. Tong Z, Zhang J, Luo W, Wang W, Li F, Li H, Luo H, Lu J, Zhou J, Wan Y, He $\mathrm{R}$. Urine formaldehyde level is inversely correlated to mini mental state examination scores in senile dementia // Neurobiol Aging. 2011 Jan;32(1):31-41. doi: 10.1016/j.neurobiolaging.2009.07.013. Epub 2009 Oct 29.

17. Ukai K, Mizuno Y. Physical complications for elderly inpatients with senile dementia in the Imaise Branch of Ichinomiya City Hospital // Psychogeriatrics. 2009 Dec;9(4):167-72. doi: 10.1111/j.1479-8301.2009.00300.x.

18. Vinters HV. Emerging concepts in Alzheimer's disease // Annu Rev Pathol. 2015;10:291-319. doi: 10.1146/annurev-pathol-020712-163927. Epub 2014 Oct 29.

19. Yan H, Li L, Tang XC. Treating senile dementia with traditional Chinese medicine // Clin Interv Aging. 2007;2(2):201-8.

\section{References}

1. Atamanov A.A. Zhurnal nevrologii i psikhiatrii im. S.S. Korsakova. M.: Media Sfera, 2008. №5. P. 19-24.

2. Vasenina E.E., Veryugina N.I., Levin O.S. Aktual'nye voprosy gerontopsikhiatrii. 2015. № 3. P. 26-28.

3. Vsemirnaya organizatsiya zdravookhraneniya. Global'nyy plan deystviy po predot-vrashcheniyu dementsiy na 2017-2025 [World Health Organization. Global Action Plan to Prevent Dementia for 2017-2025]. www.who.int/entity/ mediacentre/news/releases/2017/dementia-triple-affected/ru/

4. Gusev E.I. (ed). Nevrologiya. Natsional'noe rukovodstvo [Neuroscience. National leadership]. M.: GEOTAR-Media, 2009. $1035 \mathrm{~s}$.

5. Kamenetskaya M.I. Narushenie kognitivnykh funktsiy pri psikhicheskikh zabolevani-yakh v pozdnem vozraste [Violation of cognitive functions in mental illnesses in later life]. Tula, 2013. 50 p. P. 29-33.

6. Levin O.S. Trudnyy patsient. 2014. V.12. № 5. P. 2-6.

7. Ferstl Kh. Dementsiya / Khans Ferstl, Al'fred Melike, Klaus Vaykhel'. M.: MED-press-inform, 2011. $639 \mathrm{~s}$. 
8. Ballard C, Patel A, Oyebode F, Wilcock G. Cognitive decline in patients with Alzheimer's disease, vascular dementia and senile dementia of Lewy body type. Age Ageing. 1996 May;25(3):209-13.

9. Fukunishi I, Hosokawa K. A study of psychological aspects of families living together with senile dementia. Jpn J Psychiatry Neurol. 1990 Mar;44(1):19-24.

10. Gray AJ, Staples V, Murren K, Dhariwal A, Bentham P. Olfactory identification is impaired in clinic-based patients with vascular dementia and senile dementia of Alzheimer type. Int J Geriatr Psychiatry. 2001 May;16(5):513-7.

11. Guekht A, Skoog I, Korczyn AD, et al. A Randomised, Double- Blind, Placebo-Controlled Trial of Actovegin in Patients with Post-Stroke Cognitive Impairment: ARTEMIDA Study Design. Dement. Geriatr. Cogn. Dis. Extra. 2013. V. 3. № 1. P. 459-467.

12. Jack CR, Knopman DS, Jagust WJ, Petersen RC, et al. Tracking pathophysiological processes in Alzheimer's disease: an updated hypothetical model of dynamic biomarkers. Lancet Neurology. 2013;12(2):207-16

13. Korczyn AD. Why have we failed to cure Alzheimer's disease? J Alzheimers Dis. 2012; 29 (2): 275-82. doi: 10.3233/JAD-2011-110359.

14. Rubin EH, Kinscherf DA, Grant EA, Storandt M. The influence of major depression on clinical and psychometric assessment of senile dementia of the Alzheimer type. Am J Psychiatry. 1991 Sep;148(9):1164-71.

15. Tack GR, Choi MH, Lee SJ. Prioritizing problem features in Korean patients with senile dementia for implementation of monitoring technologies. Psychiatry Res. 2011 May 30;187(3):418-23. doi: 10.1016/j.psychres.2010.06.031. Epub 2010 Jul 24.

16. Tong Z, Zhang J, Luo W, Wang W, Li F, Li H, Luo H, Lu J, Zhou J, Wan Y, He $\mathrm{R}$. Urine formaldehyde level is inversely correlated to mini mental state examination scores in senile dementia. Neurobiol Aging. 2011 Jan;32(1):31-41. doi: 10.1016/j.neurobiolaging.2009.07.013. Epub 2009 Oct 29.

17. Ukai K, Mizuno Y. Physical complications for elderly inpatients with senile dementia in the Imaise Branch of Ichinomiya City Hospital. Psychogeriatrics. 2009 Dec;9(4):167-72. doi: 10.1111/j.1479-8301.2009.00300.x.

18. Vinters HV. Emerging concepts in Alzheimer's disease. Annu Rev Pathol. 2015;10:291-319. doi: 10.1146/annurev-pathol-020712-163927. Epub 2014 Oct 29.

19. Yan H, Li L, Tang XC. Treating senile dementia with traditional Chinese medicine. Clin Interv Aging. 2007;2(2):201-8.

\section{ДАННЫЕ ОБ АВТОРАХ}

Ефименко Татьяна Сергеевна, доцент кафедры клинической психологии и социальной работы, кандидат медицинских наук 
Южно-Уральский государственный медиџинский университет ул. Воровского, 64, г. Челябинск, Челябинская обл., 454092, Российская Федерация tomara_n@mail.ru

Локтева Дарья Николаевна, клинический психолог

Областная клиническая специализированная психоневрологическая больница №1

ул. Кузнецова, 2A, г. Челябинск, Челябинская обл., 454028, Российская Федераиия

dasha_Lokteva@list.ru

Перевощикова Анастасия Алексеевна, студент

Южно-Уральский государственный медицинский университет ул. Воровского, 64, г. Челябинск, Челябинская обл., 454092, Российская Федераиия perevoshhikova-anastasija@inbox.ru

\section{DATA ABOUT THE AUTHORS}

Efimenko Tatyana Sergeevna, Clinical Psychology and Social Work Department South-Urals State Medical University, Chelyabinsk, Russian Federation 64, Vorovsky Str., Chelyabinsk, Chelyabinsk region, 454092, Russian Federation

tomara_n@mail.ru

SPIN-code: 320105

ORCID: 0000-0003-0891-3421

Lokteva Darya Nikolaevna, Clinical Psychologist

Regional psychiatry clinics №1

2A, Kuznetsova Str., Chelyabinsk, Chelyabinsk region, 454028, Russian Federation

dasha_Lokteva@list.ru

\section{Perevoschikova Anastasia Alekseevna, Student}

South-Urals State Medical University, Chelyabinsk, Russian Federation 64, Vorovsky Str., Chelyabinsk, Chelyabinsk region, 454092, Russian Federation

perevoshhikova-anastasija@inbox.ru 